\title{
MODELAGEM NUMÉRICA DO EFEITO SISMOELÉTRICO EM MEIOS 2D
}

\author{
Francisco Vanzeler ${ }^{1}$ e Viatcheslav Priimenko ${ }^{2}$ \\ Recebido em 11 abril, 2008 / Aceito em 15 janeiro, 2009 \\ Received on April 11, 2008 / Accepted on January 15, 2009
}

\begin{abstract}
This work uses the equations of the coupled poroelastic-electromagnetic phenomena to simulate by finite-difference methods the seismoelectric responses of 2D saturated porous models. The seismic source was of explosive type with frequencies in a seismic range up to $100 \mathrm{~Hz}$. The receivers were arranged in horizontal and vertical positions. Several heterogeneous porous models were simulated to define the interface type enabling the generation of seismoelectric signals, the minimum dimension of heterogeneity that is possible to detect and the maximum investigation range of seismoelectric tools. The seismoelectric response was observed at interface of porosity, salinity, lithology and oil-water contact. These results provided a quantitative base for planning of seismoelectric acquisition jointly with surface seismic, well and interwell seismic tools for targets inside of the investigation range.
\end{abstract}

Keywords: electrical and seismic fields, electrokinetic coupling, porous saturated media, numerical modeling, finite differences.

RESUMO. Este trabalho utiliza as equações dos fenômenos poroelástico-eletromagnéticos acoplados para simular pelo método das diferenças finitas no domínio do tempo as respostas sismoelétricas de modelos porosos saturados bidimensionais. As fontes sísmicas são do tipo explosivo com freqüência de corte $100 \mathrm{~Hz}$. Os receptores foram localizados na direção horizontal e na vertical. Foram simulados cinco modelos porosos heterogêneos cujas respostas serviram para definir os tipos de interfaces que geram sinais sismoelétricos, as dimensões mínimas da heterogeneidade para detectabilidade sismoelétrica e o raio de investigação máxima. Observou-se resposta sismoelétrica irradiada em interfaces com contraste de porosidade, salinidade, litologia e fluido óleo-água. Os resultados obtidos sugerem a possibilidade de se planejar uma aquisição sismoelétrica conjuntamente com uma aquisição sísmica de superfície para objetivos rasos ou com uma aquisição sísmica em poço e interpoços para objetivos dentro do raio de investigação máxima.

Palavras-chave: campos elétrico e sísmico, acoplamento eletrocinético, meio poroso saturado, simulação numérica, diferenças finitas.

\footnotetext{
1 Petróleo Brasileiro S.A., Rua Elias Agostinho, 655, Bloco A, Sala 302, Macaé, RJ, Brasil. Tel.: (22) 2761-6965 - E-mail: joclean@petrobras.com.br

2 Laboratório de Engenharia e Exploração de Petróleo (LENEP), Universidade Estadual do Norte Fluminense Darcy Ribeiro (UENF), Rod. Amaral Peixoto, km 163, 27925-310, Macaé, RJ, Brasil. Tel.: (22) 2796-9753; Fax: (22) 2796-9734 - E-mail: slava@lenep.uenf.br
} 


\section{INTRODUÇÃo}

A propagação de ondas sísmicas em meio poroso saturado causa pequenas deformações transientes na matriz da rocha e no espaço poroso. Estas deformações perturbam a dupla camada elétrica que existe na superfície de contato sólido-fluido, e geram campos eletromagnéticos de amplitude observável. 0 interesse neste trabalho são os campos elétricos denominados de campos sismoelétricos.

0 acoplamento dos campos sísmicos com campos sismoelétricos é feito pelo coeficiente de acoplamento eletrocinético que depende da freqüência, cujo valor aumenta com 0 aumento da permissividade elétrica do fluido e do potencial zeta (potencial elétrico que aparece na dupla camada elétrica), e diminui com 0 aumento da condutividade elétrica da matriz da rocha e do fluido, e com o aumento da viscosidade do fluido. As propriedades poroelásticas do meio, como o tipo de fluido dos poros, a porosidade, a permeabilidade, e as propriedades elásticas, também afetam a conversão e a propagação do sinal sismoelétrico.

Os campos elétricos gerados pelos campos sísmicos são formados por uma combinação do campo elétrico confinado ao campo sísmico, que se propaga com a mesma velocidade do sinal sísmico, mais o campo elétrico irradiado, que se propaga com a velocidade de propagação de um campo elétrico difusivo no meio poroso.

0 campo elétrico confinado é gerado pela separação estacionária das cargas elétricas da dupla camada elétrica devido ao gradiente de pressão dentro da frente de onda sísmica, cuja amplitude depende das propriedades físicas e químicas do meio poroso saturado. 0 campo elétrico irradiado é gerado quando o campo elétrico confinado à onda sísmica atinge um contraste nas propriedades físicas e/ou químicas do meio poroso saturado, provocando uma alteração na separação estacionária das cargas elétricas da dupla camada elétrica, uma separação de cargas variável com o tempo que se irradia no meio, carregando informações sobre a posiç̧ão das descontinuidades e suas propriedades físicas e químicas.

As medidas sismoelétricas podem ser observadas nas freqüências sísmicas, sônicas e ultrasônicas, podendo ser adquiridas conjuntamente com dados sísmicos terrestres, dados sísmicos de fundo oceânico, dados sísmicos de poço, em perfilagens sônicas e em medidas ultrasônicas de laboratório.

0 potencial para utilização das medidas sismoelétricas como ferramenta geofísica vem da possibilidade de extrair informações não acessíveis ao sinal sísmico isoladamente. Tais informações incluem as propriedades químicas do fluido, a determinação da permeabilidade hidráulica, o mapeamento de descontinuidades associadas a contatos entre fluidos diferentes, a salinidades diferentes, a contatos litológicos, a contatos entre diferentes regiões permo-porosas, e a detecção direta de fluidos.

No entanto, a dificuldade imposta pelo grande número de parâmetros dos quais depende 0 sinal sismoelétrico (parâmetros poroelásticos e os parâmetros associados ao coeficiente de acoplamento eletrocinético) impede 0 uso direto da ferramenta sismoelétrica para determinar tais parâmetros sob condições reais, apesar do sucesso de alguns trabalhos experimentais (Murty, 1985; Thompson \& Gist, 1993; Butler et al., 1996; Mikhailov et al., 1997 e 2000; Zhu et al., 1999; Garambois \& Dietrich, 2001; Fourie, 2003; Zhu \& Toksöz, 2003 e 2005; Haines, 2004; Dupuis et al., 2007; Strahser et al., 2007).

É importante estudar a influência destes parâmetros isoladamente nas amplitudes sismoelétricas para se ter controle das respostas através da análise quantitativa. Para este estudo controlado a modelagem numérica das equações sismoelétricas aplicada a modelos conhecidos ajudará na previsão do comportamento do sinal sismoelétrico em situações reais.

Este trabalho se propõe estudar quantitativamente a influência de alguns destes parâmetros na amplitude do sinal sismoelétrico. Para alcançar este objetivo, na primeira etapa, resolvemos numericamente pelo método das diferenças finitas no domínio do tempo, o caso sismoelétrico 2D das equações que governam 0 acoplamento poroelástico-eletromagnético nas freqüências sísmicas. Na segunda etapa, aplicamos a solução numérica em modelos de rochas siliciclásticas saturadas com água ou óleo, para estudar respostas sismoelétricas em poços e na superfície aos contrastes de porosidade, salinidade, contato óleo-água, contato argila-areia, e estudar modelos de detectabilidade de uma camada fina de argila, e um pequeno bloco de argila. E na terceira etapa analisamos os resultados das respostas sismoelétricas para entender e controlar aspectos relevantes das respostas dos modelos úteis para desenvolvimento de projetos de aquisição e interpretação de dados sismoelétricos em conjunto com dados sísmicos.

A teoria moderna da interação eletrocinética foi desenvolvida por Pride (Pride, 1994) e são escritas no domínio da freqüência temporal $\omega$ como:

$$
\begin{gathered}
\nabla \cdot \boldsymbol{\tau}=-i \omega\left(\rho \dot{\mathbf{u}}+\rho_{f} \dot{\mathbf{w}}\right), \\
-\nabla P_{f}=-i \omega\left(m \dot{\mathbf{w}}+\rho_{f} \dot{\mathbf{u}}\right)+\frac{\eta}{k(\omega)} \dot{\mathbf{w}}, \\
\boldsymbol{\tau}=\left(\lambda_{c} \nabla \cdot \mathbf{u}+\alpha M \nabla \cdot \mathbf{w}\right) \mathbf{I}+\mu\left(\nabla \mathbf{u}+\nabla \mathbf{u}^{T}\right),
\end{gathered}
$$




$$
\begin{gathered}
-P_{f}=\alpha M \nabla \cdot \mathbf{u}+M \nabla \cdot \mathbf{w}, \\
\dot{\mathbf{w}}=L(\omega) \mathbf{E}-\frac{k(\omega)}{\eta}\left(\nabla P_{f}+i \omega \rho_{f} \dot{\mathbf{u}}\right), \\
\mathbf{J}=\sigma(\omega) \mathbf{E}-L(\omega)\left(\nabla P_{f}+i \omega \rho_{f} \dot{\mathbf{u}}\right), \\
\nabla x \mathbf{E}=i \omega \mathbf{B}, \\
\nabla x \mathbf{H}=-i \omega \mathbf{D}+\mathbf{J}, \\
\mathbf{D}=\varepsilon_{0}\left[\frac{\varphi}{\alpha_{\infty}}\left(k_{f}-k_{s}\right)+k_{s}\right] \mathbf{E}, \\
\mathbf{B}=\mu_{0} \mathbf{H},
\end{gathered}
$$

onde $\nabla=\left(\partial_{1}, \partial_{2}, \partial_{3}\right): \partial_{1}=\partial / \partial_{x}, \partial_{2}=\partial / \partial_{y}, \partial_{3}=$ $\partial / \partial_{z} ; \boldsymbol{\tau}$ é o campo de tensão volumétrico; $P_{f}$ é a pressão do fluido; u é o campo de deslocamento no sólido; w é o campo de deslocamento relativo entre 0 sólido e o fluido; I é 0 tensor de identidade; $\rho$ é a densidade volumétrica; $\rho_{f}$ é a densidade do fluido; $m$ é a densidade equivalente que representa a massa induzida devido à oscilação do fluido no sólido, ou devido à transferência de energia cinética entre as fases; $\lambda_{c}$ e $\mu$ são as constantes de Lamé do sólido; $\alpha M$ e $M$ são os módulos de Biot do meio; $k(\omega)$ é a permeabilidade hidráulica; $\eta$ é a viscosidade do fluido; $L(\omega)$ é 0 coeficiente de acoplamento eletrocinético; J é a densidade de corrente elétrica; $\sigma(\omega)$ é a condutividade elétrica; E é o campo elétrico; D é o campo de deslocamento elétrico; H é o campo magnético; $\mathbf{B}$ é 0 campo de indução magnética; $\varepsilon_{0}$ é a permissividade elétrica do vácuo; $k_{f}$ e $k_{s}$ são as permissividades relativas do fluido e do sólido; $\varphi$ é a porosidade; $\alpha_{\infty}$ é a tortuosidade do meio poroso; $\mu_{0}$ é a permeabilidade magnética do vácuo.

0 acoplamento dos campos sísmicos com os campos eletromagnéticos é feito essencialmente pelo coeficiente de acoplamento eletrocinético $L(\omega)$, pois quando seu valor é zero, as Eqs. (1)-(5) de Biot e as Eqs. (6)-(10) de Maxwell ficam desacopladas. 0 valor do coeficiente de acoplamento eletrocinético é diretamente proporcional à permissividade elétrica do fluido e ao potencial zeta, e inversamente proporcional à condutividade elétrica e a viscosidade do fluido.

\section{EQUAÇÕES SISMOELÉTRICAS 2D}

0 sistema (1)-(10), denominado o sistema de Pride, foi desenvolvido pela primeira vez por Pride (Pride, 1994), usando a técnica da média num volume finito, ou seja, parte da escala microscópica para a escala macroscópica.

Neste trabalho vamos considerar somente 0 caso em que a fonte de perturbação do meio poroso é de origem mecânica, e recebem a denominação de fenômenos sismoelétricos. Isto significa que nem todos os termos nas equações sismoelétricas são necessários para serem incluídas.

$\mathrm{Na}$ Eq. (8) 0 termo associado à corrente de deslocamento dielétrica, $-i \omega \mathbf{D}$, pode ser negligenciado em relação à corrente de condução, $\mathbf{J}$, devido à razão

$$
\frac{\omega \varepsilon}{\sigma}<10^{-4}
$$

estar situada na faixa difusiva para a freqüência $10^{-4} \mathrm{em}$ toda banda sísmica, e para a condutividade $k$ do meio poroso de interesse (rochas sedimentares).

Outro termo negligenciado é o termo $L(\omega) \mathbf{E}$ associado à eletro-osmose na Eq. (5). A amplitude deste efeito é calculada considerando o campo elétrico gerado pela onda compressional propagando através de um meio poroso homogêneo. Neste caso, a acumulação de carga nos picos e vales da onda gera um campo elétrico que gera uma corrente elétrica que é compensada pela corrente de streaming tal que $\mathbf{J}=\mathbf{0}$ na Eq. (6), e 0 campo elétrico será:

$$
\mathbf{E}=\frac{L(\omega)}{\sigma}\left(\nabla P_{f}+i \omega \rho_{f} \dot{\mathbf{u}}\right) .
$$

Substituindo a Eq. (12) na Eq. (5) obtemos:

$$
-\dot{\mathbf{w}}=\frac{k}{\eta}\left(1-\frac{\eta L^{2}}{k \sigma}\right)\left(\nabla P_{f}+i \omega \rho_{f} \dot{\mathbf{u}}\right) .
$$

0 termo adimensional $\eta L^{2} / k \sigma$ representa a retroalimentação eletro-osmótica, que para o meio poroso de interesse neste trabalho que são as rochas sedimentares, 0 valor típico:

$$
\frac{\eta L^{2}}{k \sigma}<10^{-5}
$$

que pode ser negligenciado em relação à unidade. Isto significa que a perturbação eletro-osmótica $L(\omega) \mathbf{E}$ na Eq. (5) é uma minúscula perturbação tal que o fluxo de fluido induzido pelo campo elétrico gerado pela onda mecânica é desprezível. A desconsideração deste termo implica que a resposta poroelástica se desacopla da resposta eletromagnética (o caso contrário continua acoplado). Desta forma, substituindo a condição (14) na Eq. (13), obtemos:

$$
-\dot{\mathbf{w}}=\frac{k}{\eta}\left(\nabla P_{f}+i \omega \rho_{f} \dot{\mathbf{u}}\right) .
$$

Baseado na Eq. (15) podemos calcular a densidade de corrente associado à perturbação mecânica. Para isso, substituímos na Eq. (6) a Eq. (15), e obtemos:

$$
\mathbf{J}=\frac{\eta L(\omega)}{k} \dot{\mathbf{w}}+\sigma \mathbf{E} .
$$


Outra restrição imposta às equações de Pride para o caso sismoelétrico está ligada à razão entre a profundidade de investigação e o efeito de superfície em condutores (skin effect - tendência de correntes alternadas fluírem próximo a superfície dos condutores e a se atenuarem fortemente com a profundidade, numa espécie de aumento da impedância elétrica com a profundidade, de tal forma que a profundidade em que as correntes foram atenuadas em um neper, denominamos de skin depth). 0 efeito de skin depth é calculado da seguinte forma:

$$
\delta=\frac{1}{\sqrt{\omega \mu \sigma}} .
$$

Nas freqüências sísmicas ( $f<1 \mathrm{kHz}$ ), e para 0 meio poroso das rochas sedimentares, 0 valor do skin depth será $\delta>1 \mathrm{~km}$. Como 0 sinal sismoelétrico já foi detectado em distâncias da ordem de centenas de metros, (Thompson \& Gist, 1993), a razão profundidade $d$ e 0 skin depth $\delta$ é muito menor que um, $\frac{d}{\delta}<<$ 1. Isto significa que os campos eletromagnéticos difusivos ditados pela condição (11), são quase estáticos, ou seja, os efeitos de indução na Eq. (7) podem ser negligenciados, ou melhor:

$$
\nabla \times \mathbf{E}=\mathbf{0} .
$$

Assim, os campos elétricos podem ser derivados de uma função $\Phi$ chamada potencial elétrico da seguinte forma:

$$
\mathbf{E}=-\nabla \Phi .
$$

Substituindo a Eq. (19) na Eq. (16) e o resultado obtido substituído na Eq. (8) e, então calcula-se a divergência, obtendo-se:

$$
\nabla \cdot(\sigma \nabla \Phi)=\nabla \cdot\left(\frac{\eta L}{k} \dot{\mathbf{w}}\right) .
$$

A Eq. (20) é a equação de Poisson para potencial elétrico $\Phi$. Para se calcular o campo elétrico, primeiro resolvemos a Eq. (20) para o potencial elétrico, e, em seguida calculamos 0 gradiente do potencial elétrico na Eq. (19). 0 campo magnético associado é da ordem de pico Tesla (Haines, 2004) e muito pequeno para ser detectado em experimentos de campo.

Considerando o caso 2D no plano XZ e usando as Eqs. (19) e (20), mais as definições

$$
\begin{aligned}
& \mathbf{U}=\left(U_{1}, U_{3}\right) \equiv\left(\dot{u_{1}}, \dot{u_{3}}\right), \\
& \mathbf{W}=\left(W_{1}, W_{3}\right) \equiv\left(\dot{w}_{1}, \dot{w}_{3}\right), \\
& \tau=\left(\begin{array}{ll}
\tau_{11} & \tau_{13} \\
\tau_{31} & \tau_{33}
\end{array}\right), \tau_{13}=\tau_{31},
\end{aligned}
$$

e devido ao efeito de relaxação causado pelos mecanismos de atenuação serem desconsiderados nas freqüências sísmicas (Pride, 1994), os coeficientes do nosso sistema não dependem da freqüência temporal $\omega$, podemos reescrever 0 sistema (1)-(10) no domínio temporal como:

$$
\begin{gathered}
\left(m \rho-\rho_{f}^{2}\right) \partial_{t} \mathbf{U}=m \nabla \cdot \tau+\rho_{f} \nabla P_{f}+\rho_{f} \frac{\eta}{k} \mathbf{W}, \\
\left(\rho_{f}^{2}-m \rho\right) \partial_{t} \mathbf{W}=\rho_{f} \nabla \cdot \tau+\rho \nabla P_{f}+\rho \frac{\eta}{k} \mathbf{W}, \\
\partial_{t} \boldsymbol{\tau}=\left(\lambda_{c} \nabla \cdot \mathbf{U}+\alpha M \nabla \cdot \mathbf{W}\right) \mathbf{I}+\mu\left(\nabla \mathbf{U}+\nabla \mathbf{U}^{T}\right), \\
\partial_{t} P_{f}=\alpha M \nabla \cdot \mathbf{U}+M \nabla \cdot \mathbf{W}, \\
\nabla \cdot(\sigma \nabla \Phi)=\nabla \cdot\left(\frac{\eta L}{k} \mathbf{W}\right), \\
\mathbf{E}=-\nabla \Phi
\end{gathered}
$$

onde $\nabla=\left(\partial_{1}, \partial_{3}\right)$.

\section{ESQUEMA NUMÉRICO PROPOSTO}

A implementação convencional das Eqs. (21)-(24), veja (André, 2005), mostra que quando a razão entre a viscosidade e a permeabilidade é muito elevada, ou seja, $\eta / k>10^{7}$, foi constatado que as soluções tornam-se instáveis numericamente. 0 tratamento desta instabilidade foi a divisão do intervalo temporal, fato que aumenta de sobremaneira o tempo de processamento. Uma conseqüência desta restrição é que, nos casos reais, os valores da viscosidade $\eta$ estão entre $10^{-4}$ e 1 Pa.s, e os valores de $k$ estão entre $10^{-20}$ a $10^{-10} \mathrm{~m}^{2}$, implicando que na maioria destes casos teríamos instabilidade numérica. A solução deste problema foi apresentada em (Carcione \& Quiroga-Goode, 1995; Carcione, 2001), e que consiste em resolver separadamente o problema numa parte analítica e numa parte numérica da seguinte forma.

Dividindo (21) por $\left(m \rho-\rho_{f}^{2}\right)$, e (22) por $\left(\rho_{f}^{2}-m \rho\right)$, podemos reescrever as Eqs. (21)-(24) em termos do vetor

$$
\mathbf{Y}=\left(U_{1}, U_{3}, W_{1}, W_{3}, \tau_{11}, \tau_{33}, \tau_{13}, P_{f}\right)^{T}
$$

na forma matricial:

$$
\partial_{t} \mathbf{Y}=\mathbf{A} \cdot \mathbf{Y},
$$


onde

$$
\mathbf{A}=\left(\begin{array}{cccccccc}
0 & 0 & L a & 0 & L m \partial_{1} & 0 & L m \partial_{3} & L \rho_{f} \partial_{1} \\
0 & 0 & 0 & L a & 0 & L m \partial_{3} & L m \partial_{1} & L \rho_{f} \partial_{3} \\
0 & 0 & S b & 0 & S \rho_{f} \partial_{1} & 0 & S \rho_{f} \partial_{3} & S \rho \partial_{1} \\
0 & 0 & 0 & S b & 0 & S \rho_{f} \partial_{3} & S \rho_{f} \partial_{1} & S \rho \partial_{3} \\
A \partial_{1} & B \partial_{3} & C \partial_{1} & C \partial_{3} & 0 & 0 & 0 & 0 \\
B \partial_{1} & A \partial_{3} & C \partial_{1} & C \partial_{3} & 0 & 0 & 0 & 0 \\
\mu \partial_{3} & \mu \partial_{1} & 0 & 0 & 0 & 0 & 0 & 0 \\
-C \partial_{1} & -C \partial_{3} & -M \partial_{1} & -M \partial_{3} & 0 & 0 & 0 & 0
\end{array}\right)
$$

Os coeficientes da matriz A são definidos como:

$$
a=\frac{\rho_{f} \eta}{k}, \quad b=\frac{\rho \eta}{k}, A=\lambda_{c}+2 \mu, B=\lambda_{c}, C=\alpha M, L=-S=\frac{1}{m \rho-\rho_{f}^{2}} .
$$

Uma avaliação dos valores numéricos dos termos da matriz A feita em (Santos et al., 2006) mostra que os valores extremamente altos dos termos que contém a razão entre a viscosidade $\eta$ sobre a permeabilidade $k$ quando comparados aos outros termos da matriz são os responsáveis pela instabilidade. A solução consiste em resolver separadamente o sistema (27)-(28) em dois subsistemas, o primeiro subsistema contendo os valores altos e resolvidos analiticamente, escrito da seguinte forma

$$
\partial_{t} \mathbf{Y}=\mathbf{A}_{1} \cdot \mathbf{Y}
$$

com matriz $\mathbf{A}_{1}$ definida como

$$
\mathbf{A}_{1}=\left(\begin{array}{cccccccc}
0 & 0 & L a & 0 & 0 & 0 & 0 & 0 \\
0 & 0 & 0 & L a & 0 & 0 & 0 & 0 \\
0 & 0 & S b & 0 & 0 & 0 & 0 & 0 \\
0 & 0 & 0 & S b & 0 & 0 & 0 & 0 \\
0 & 0 & 0 & 0 & 0 & 0 & 0 & 0 \\
0 & 0 & 0 & 0 & 0 & 0 & 0 & 0 \\
0 & 0 & 0 & 0 & 0 & 0 & 0 & 0
\end{array}\right)
$$

cuja solução é obtida analiticamente da seguinte forma:

$$
\begin{gathered}
\mathbf{W}^{n+1}=\mathbf{W}^{n} e^{S b \Delta t}, \\
\mathbf{U}^{n+1}=\mathbf{U}^{n}-\frac{\rho_{f}}{\rho}\left(e^{S b \Delta t}-1\right) \mathbf{W}^{n},
\end{gathered}
$$

onde 0 índice $n+1$ indica 0 tempo atual, $n 0$ tempo anterior, e $\Delta t 0$ intervalo temporal de discretização.

0 segundo subsistema contendo os valores menores e resolvidos numericamente por diferenças finitas, escrito da seguinte forma:

$$
\partial_{t} \mathbf{Y}=\mathbf{A}_{2} \mathbf{Y}
$$

onde matriz $\mathbf{A}_{2}$ definida como

$$
\mathbf{A}_{2}=\left(\begin{array}{cccccccc}
0 & 0 & 0 & 0 & L m \partial_{1} & 0 & L m \partial_{3} & L \rho_{f} \partial_{1} \\
0 & 0 & 0 & 0 & 0 & L m \partial_{3} & L m \partial_{1} & L \rho_{f} \partial_{3} \\
0 & 0 & 0 & 0 & S \rho_{f} \partial_{1} & 0 & S \rho_{f} \partial_{3} & S \rho \partial_{1} \\
0 & 0 & 0 & 0 & 0 & S \rho_{f} \partial_{3} & S \rho_{f} \partial_{1} & S \rho \partial_{3} \\
A \partial_{1} & B \partial_{3} & C \partial_{1} & C \partial_{3} & 0 & 0 & 0 & 0 \\
B \partial_{1} & A \partial_{3} & C \partial_{1} & C \partial_{3} & 0 & 0 & 0 & 0 \\
\mu \partial_{3} & \mu \partial_{1} & 0 & 0 & 0 & 0 & 0 & 0 \\
-C \partial_{1} & -C \partial_{3} & -M \partial_{1} & -M \partial_{3} & 0 & 0 & 0 & 0
\end{array}\right)
$$


A solução do segundo subsistema obtida numericamente no tempo $n$ servirá de entrada para a solução analítica (32)(33) do tempo $n+1$. Desta forma, podemos simular modelos poroelásticos com altos valores dos contrastes de viscosidadepermeabilidade.

As variáveis e parâmetros que aparecem nas Eqs. (21)(26) sismoelétricas foram distribuídas espacialmente na maIha intercalada, veja (Mandariaga, 1976; Virieux, 1984, 1986) encaixada na célula bidimensional eletromagnética, veja (Yee, 1966). Nas equações poroelásticas, as derivadas espaciais usaram aproximações de quarta ordem e as derivadas temporais usaram aproximações de segunda ordem baseados em (Levander, 1988):

$$
\begin{aligned}
u_{x}= & \frac{1}{h}\left\{\frac{9}{8}\left[u\left(x+\frac{h}{2}\right)-u\left(x-\frac{h}{2}\right)\right]\right. \\
& \left.-\frac{1}{24}\left[u\left(x+\frac{3 h}{2}\right)-u\left(x-\frac{3 h}{2}\right)\right]\right\}+O\left(h^{4}\right), \\
u_{t}= & \frac{u\left(t+\frac{\Delta t}{2}\right)-u\left(t-\frac{\Delta t}{2}\right)}{\Delta t}+O\left((\Delta t)^{2}\right) .
\end{aligned}
$$

Nas equações eletromagnéticas quase-estáticas, as derivadas espaciais da equação de Poisson e do gradiente do potencial elétrico, usaram aproximações de segunda ordem baseados em (Cuminato \& Meneguette Jr., 2002):

$$
\begin{gathered}
u_{x x}=\frac{u(x+h)-2 u(x)+u(x-h)}{h^{2}}+O\left(h^{2}\right), \\
u_{x}=\frac{u(x+h)-u(x-h)}{2 h}+O\left(h^{2}\right) .
\end{gathered}
$$

Em virtude da complexidade não mostramos o esquema construído.

Na Figura 1 mostramos as posições das variáveis e parâmetros sismoelétricos dentro da célula básica.

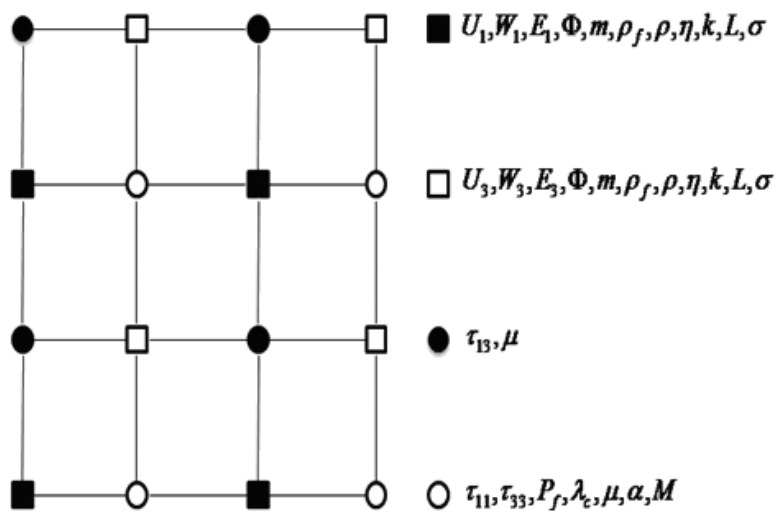

Figura 1 - Célula espacial sismoelétrica com as posições das variáveis e parâmetros.
A dispersão e estabilidade numérica foram controladas pelas seguintes condições:

$$
h<\frac{V_{\min }}{5 f_{\max }}, \quad \Delta t<\frac{h}{5 V_{\max }} .
$$

Para as condições (36) o espaçamento da malha foi de $5.0 \mathrm{~m}$, e 0 intervalo de amostragem foi de $0.4 \mathrm{~ms}$.

Adotou-se as condições de contorno desenvolvidas em (Cerjan et al., 1985) para atenuação de bordas da malha numérica. Este método se baseia na redução gradual das amplitudes numa faixa de pontos nos contornos da fronteira da malha numérica. Ou seja, uma zona de transição de atenuação que varia suavemente de uma região interna para uma região externa como um filtro espacial, cuja expressão matemática unidirecional é da seguinte forma:

$$
\Psi(i)=\Psi_{0}(i) \exp (-g(N a-i))^{2},
$$

onde $g$ é um fator de atenuação e $N a$ é o número de pontos da borda da esponja absorvente.

A fonte sísmica utilizada neste trabalho é uma função analítica do tipo explosiva (compressional), descrita pela derivada segunda da função Gaussiana, dada pela seguinte expressão:

$$
S(t)=\left(2 \pi^{2}\left(t_{d} f_{c}\right)^{2}-1\right) \exp \left(-\pi^{3}\left(t_{d} f_{c}\right)^{2}\right),
$$

onde a freqüência central $f_{c}$, é definida como:

$$
f_{c}=\frac{f_{\text {corte }}}{3 \sqrt{\pi}},
$$

$t_{d}$ é 0 tempo defasado, associado à translação temporal:

$$
t_{d}=t-\frac{2 \sqrt{\pi}}{f_{c}} .
$$

A Figura 2 apresenta a função (38) para freqüência de corte em $100 \mathrm{~Hz}$.

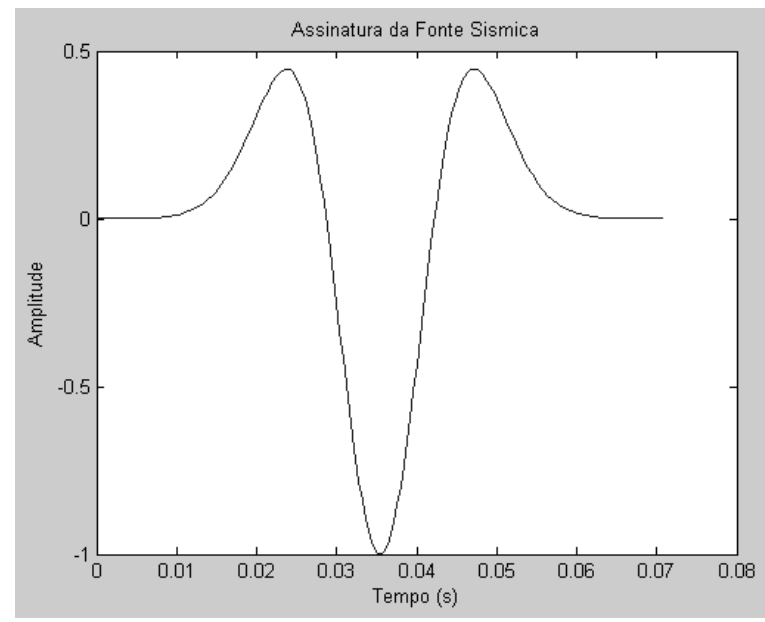

Figura 2 - Assinatura sísmica explosiva do tipo segunda derivada da função Gaussiana. 
A fonte sísmica é implementada no algoritmo através da adição da função $S(t)$ às componentes normais da tensão total $\tau_{11}$ e $\tau_{33}$ ponderada pela fração sólida, $(1-\varphi)$, e através da adição da função $S(t)$ à pressão do fluido ponderado pela fração fluida, $\varphi$, aplicada no ponto da malha em que esta localizada a fonte.

As equações sismoelétricas 2D foram codificadas usando 0 aplicativo MATLAB.

\section{MODELAGEM NUMÉRICA}

Os modelos sismoelétricos simulados computacionalmente foram construídos baseados em dados encontrados na literatura (Pride \& Morgan, 1991; Haines, 2004), e usa como meio poroso as rochas do tipo argila e arenito dispostas em geometrias que simulem as situações geológicas de interesse. As respostas sismoelétricas dos modelos investigados estarão situadas em geometrias de aquisição horizontal e vertical visando simular aquisição na superfície da Terra, no fundo oceânico e em poços. Os principais parâmetros do modelo sismoelétrico foram variados de maneira a determinar as condições controladas da detectabilidade dos sinais sismoelétricos. A amplitude do sinal sismoelétrico depende dos fatores físicos que contribuem para a movimentação das cargas elétricas da dupla camada elétrica. Desta forma, a amplitude do sinal sismoelétrico depende da amplitude sísmica (que por sua vez dependem das constantes elásticas) (Butler et al., 1999), da permeabilidade hidráulica e do fator de acoplamento eletrocinético. 0 sinal sismoelétrico é dependente da freqüência de tal forma que existe uma freqüência de transição que separa o comportamento viscoso das baixas freqüências do comportamento inercial das altas freqüências, cujo valor típico para solos e rochas sedimentares é $10^{5} \mathrm{~Hz}$, calculado como:

$$
\omega=\frac{\varphi \eta}{\alpha_{\infty} k \rho_{f}},
$$

onde $\varphi$ é a porosidade, $\eta$ é a viscosidade do fluido, $\rho_{f}$ é a densidade do fluido, $\alpha_{\infty}$ é a tortuosidade, e $k$ a permeabilidade hidráulica.

0 sinal sismoelétrico é medido em eletrodos dispostos em poços ou na superfície, conjuntamente com o sinal sísmico, medido em geofones. A amplitude típica do sinal sismoelétrico estacionário é de $0.1 \mathrm{mV}$, e a amplitude típica do sinal sismoelétrico irradiado nas interfaces é de $0.001 \mathrm{mV}$ (Haines, 2004).

Os modelos sismoelétricos simulados foram desenvolvidos a partir do arenito e do folhelho apresentado por Haines (2004), apresentados na Tabela 1.
Tabela 1 - Parâmetros das rochas porosas usados como base para construção dos modelos sismoelétricos, medidos no sistema SI.

\begin{tabular}{|c|c|c|}
\hline & Arenito & Argila \\
\hline$V_{p}$ & 1860 & 2300 \\
\hline$V_{S}$ & 1602 & 1982 \\
\hline$\rho$ & 2600 & 2600 \\
\hline$K_{S}$ & $35 \times 10^{9}$ & $25 \times 10^{9}$ \\
\hline$\varphi$ & 0.3 & 0.1 \\
\hline$k$ & $10^{-11}$ & $10^{-16}$ \\
\hline$\sigma$ & 0.01 & 0.05 \\
\hline
\end{tabular}

Os parâmetros poroelásticos foram determinados da seguinte forma:

$$
\begin{aligned}
& \lambda_{c}=K_{c}-\frac{2}{3} \mu, K_{c}=(1-\varphi) K_{s}+\varphi K_{f}, \\
& K_{s}=\rho_{s} V_{P-s}^{2}-\frac{4}{3} \rho_{s} V_{s}^{2}, \quad K_{f}=\rho_{f} V_{p-f}^{2}, \\
& \rho=\rho_{f} \varphi+\rho_{s}(1-\varphi), \rho=\rho_{f} \varphi+\rho_{s}(1-\varphi), \\
& \mu=\rho V_{s}^{2}, \quad M=\left[\frac{\varphi}{K_{f}}+\frac{(\alpha-\varphi)}{K_{s}}\right]^{-1}, \\
& \alpha=1-\frac{K}{K_{s}}, \quad K=(1-\varphi) K_{s}, C=\alpha M,
\end{aligned}
$$

onde $K_{c}$ é a compressibilidade do volume poroso, $K_{s}$ é a compressibilidade do arcabouço sólido, $K_{f}$ é a compressibilidade do fluido dos poros, $V_{p-s}$ é a velocidade compressional no arcabouço sólido, $V_{p-f}$ é a velocidade compressional no fluido, $V_{s}$ é a velocidade cisalhante.

A massa induzida que aparece devido à oscilação da fase fluida no esqueleto sólido, ou melhor, devido à transferência de energia cinética entre as fases, calculada como:

$$
m=a \frac{\rho_{f}}{\varphi} \operatorname{com} a=1-r\left(1-\frac{1}{\varphi}\right),
$$

onde $r$ é um fator associado à geometria do arcabouço (Berryman, 1980), e neste trabalho, o seu valor é igual a 0.5 .

0 coeficiente de acoplamento eletrocinético que aparece na Eq. (25) depende da freqüência e é diretamente proporcional à permissividade elétrica do fluido, $\varepsilon_{f}$, e ao potencial zeta, $\zeta$, e inversamente proporcional ao fator de formação do meio poroso saturado, $F$, e a viscosidade do fluido, $\eta$, escrito da seguinte maneira:

$$
L=\frac{\varepsilon_{f} \zeta}{\eta F},
$$

onde 0 fator de formação é definido como $F=\frac{\sigma_{f}}{\sigma}$ e $\sigma_{f}$ é a condutividade elétrica do fluido, $\sigma$ é a condutividade elétrica do meio poroso. 0 fator de formação deste trabalho usou 0 
critério adotado por Haines (2004), calculado da porosidade, da seguinte forma:

$$
F=\varphi^{m},
$$

onde $m$ é 0 fator de cimentação, cujo valor assumido neste trabalho é igual a 2.

Para o potencial zeta foi usado o modelo obtido de medidas de laboratório feitas sobre um arenito (Pride \& Morgan, 1991), escrito da seguinte forma:

$$
\zeta=0.001+0.0251 \log _{10} C_{m},
$$

onde $C_{m}$ é a concentração de sal em moles/itro. 0 potencial zeta é o principal parâmetro do acoplamento eletrocinético, pois quando se anula, a sísmica se desacopla do eletromagnetismo. 0 fluido usado em todos os modelos sintéticos foi a água.

A condutividade elétrica do fluido em solução é função da concentração de $\mathrm{NaCl}$, escrito da seguinte forma:

$$
\sigma_{f}=10 C_{m} .
$$

A condutividade da rocha saturada ou do esqueleto poroso saturado é dada por:

$$
\sigma=\frac{\sigma_{f}}{F} .
$$

As propriedades físicas dos fluidos que preenchem os espaços porosos dos modelos sismoelétricos estão resumidas na Tabela 2.

Tabela 2 - Parâmetros dos fluidos do espaço poroso usados nos modelos sismoelétricos medidos no sistema SI.

\begin{tabular}{|c|c|c|}
\hline & Água & Óleo \\
\hline $\mathrm{V}$ & 1500 & 1200 \\
\hline$\eta$ & 0.001 & 0.1 \\
\hline$\rho$ & 1000 & 800 \\
\hline $\mathrm{K}$ & $10^{9}$ & $25 \times 10^{9}$ \\
\hline$\varepsilon$ & $80 \varepsilon_{0}$ & $2 \varepsilon_{0}$ \\
\hline$\sigma$ & 0.005 & 0.00001 \\
\hline
\end{tabular}

A Figura 3 representa os modelos heterogêneos usados para simular os contrastes sismoelétricos, as posições de fonte e receptores. A primeira figura representa a geometria de aquisição sismoelétrica com receptores dispostos na horizontal simula a aquisição em superfície, e a segunda representa uma aquisição em poço. A razão pelo qual a fonte e os receptores não foram colocados na superfície do modelo horizontal foi para evitar que não houvesse superposição entre os sinais sismoelétrico confinado e irradiado.

Foram elaborados os seguintes modelos sismoelétricos 2D:

\section{Modelo de contraste de porosidade}

A simulação deste modelo visa investigar a influência do contraste de porosidade baseando-se em parâmetros que dependem da mesma como a tortuosidade (Berryman, 1980); o módulo elástico do arcabouço sólido e o módulo de elasticidade cisalhante (Pride et al., 2002); as relações entre a porosidade e a permeabilidade para arenito definido pela equação de Kozeny-Carman, discutida em (Mavko et al., 1998); as relações de Wyllie (Wyllie et al., 1956) entre a velocidade compressional efetiva volumétrica e as velocidades compressionais do fluido e do grão sólido; as relações entre a densidade efetiva volumétrica e as densidades do fluido e grão sólido (Mavko et al., 1998); as relações entre a condutividade elétrica volumétrica e a condutividade elétrica da água que satura os poros, dadas pela lei empírica de Archie; as relações entre a permissividade dielétrica volumétrica e as permissividades elétricas do fluido e do grão. Variou-se a porosidade do arenito da Tabela 1 para os valores $\varphi=0.3$ e $\varphi=0.1$ e os demais parâmetros dependentes da porosidade conforme as regras empíricas definidas. 0 fluido usado na saturação dos poros foi a

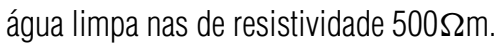

A Figura 4 mostra as respostas sísmica (Vx e Vz) e sismoelétrica (Ex e Ez) medidas nas direções $X$ e Z para 0 contraste de porosidade, com geometria de fonte e receptores baseada na Figura 3a. Observamos nesta figura um campo elétrico confinado caracterizado pelo tempo de chegada idêntico ao tempo de chegada do sinal sísmico e sem superposição com o campo elétrico irradiado com tempo de chegada aproximadamente $0.05 \mathrm{~s}$ simultâneo em todos os canais receptores Ex e Ez. As mudanças de fase simétricas em relação à posição da fonte observadas nos sinais sísmicos (Vx) e sismoelétricos (Ex) medidos na direção X estão consistentes com a polaridade da fonte sísmica. As amplitudes dos campos sismoelétricos da ordem de microvolts por metro são valores mensuráveis.

A Figura 5 mostra as respostas sísmica ( $\mathrm{Vx}$ e $\mathrm{Vz})$ e sismoelétrica ( $E x$ e Ez) medidas nas direções X e Z para o contraste de porosidade, com geometria de fonte e receptores baseada na Figura 3b. Observamos nesta figura um campo elétrico confinado caracterizado pelo tempo de chegada idêntico ao tempo de chegada do sinal sísmico superposto ao campo elétrico irradiado com tempo de chegada aproximadamente 0.07 s simultâneo em todos os canais receptores Ex e Ez. As mudanças de fase simétricas em relação à interface observadas no sinal sismoelétricos medidos na direção Z estão consistentes com a polaridade do dipolo elétrico gerado na interface. As amplitudes dos campos sismoelétricos da ordem de microvolts por metro são valores mensuráveis. 


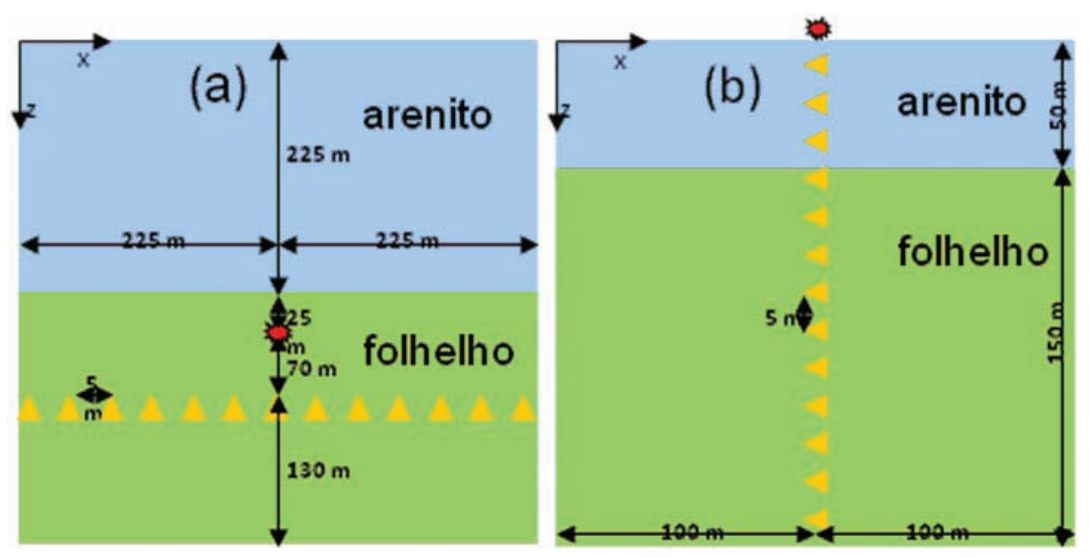

Figura 3 - Geometrias de fonte e receptores usadas nos modelos heterogêneos. A figura (a) simula receptores na horizontal e a figura (b) na vertical.

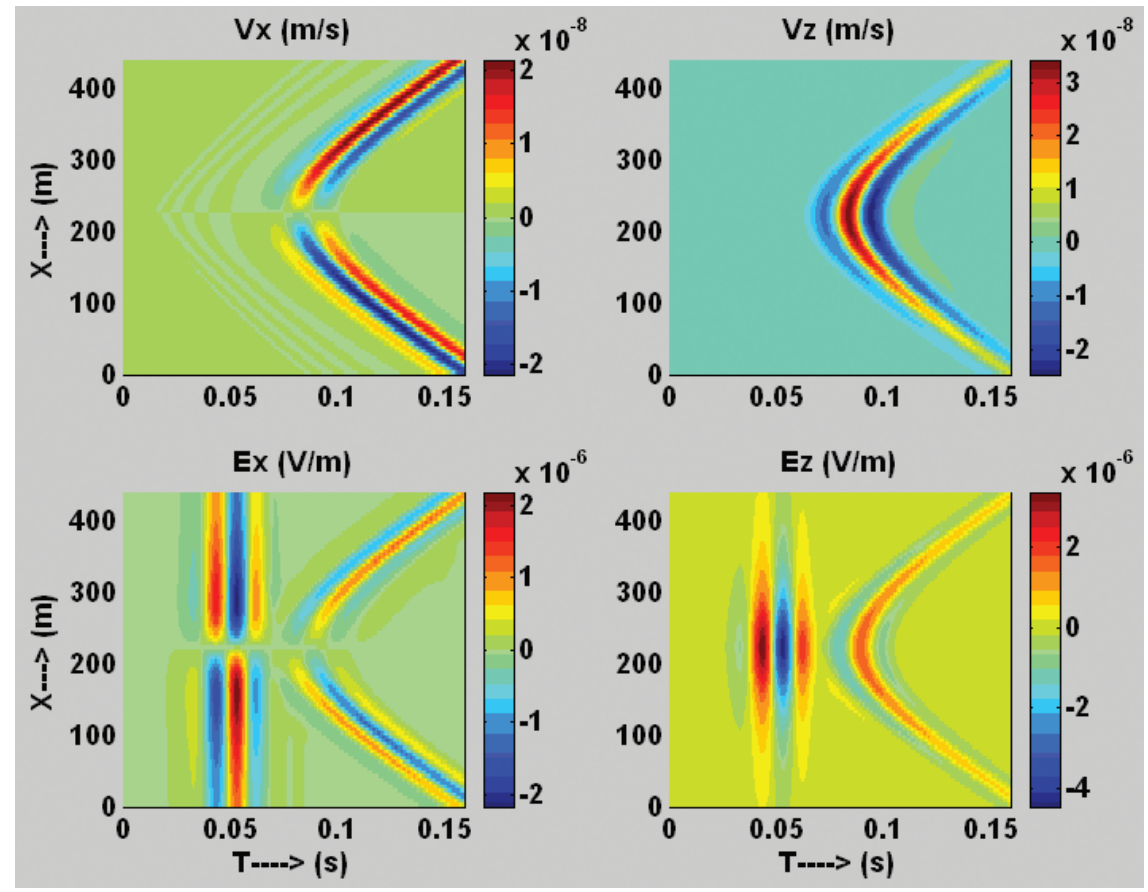

Figura 4 - Respostas sísmica (Vxe Vz) e sismoelétrica confinada-irradiada (Ex e Ez) devido ao contraste de porosidade, medidas na horizontal nas direções $X$ e $Z$.

Nos sismogramas aparece uma reflexão sísmica muito fraca no tempo de $0.08 \mathrm{~s}$.

Os resultados apresentados nas Figuras 4 e 5 confirmam que um contraste de $10 \%$ e $30 \%$ na porosidade, comum em situações reais, pode ser perfeitamente mapeado com amplitudes mensuráveis de campo elétrico irradiado. Apesar do sinal sismoelétrico em situações reais ser influenciado por todos os parâmetros, a obtenção da resposta isolada do contraste de porosidade indica a possibilidade do mapeamento de interfaces po- rosas com uso de informações adicionais, por exemplo, a fraca reflexão sísmica ao contraste de porosidade, que permitam conhecer os efeitos dos outros parâmetros.

\section{Modelo de contraste de salinidade}

Este modelo tem por base 0 trabalho (Pride \& Morgan, 1991) onde, baseado em experiências, descreve 0 potencial zeta em função da salinidade (veja a Eq. (43)). Sabe-se que a contaminação de aqüíferos pode ser medida pelo aumento do índice de 


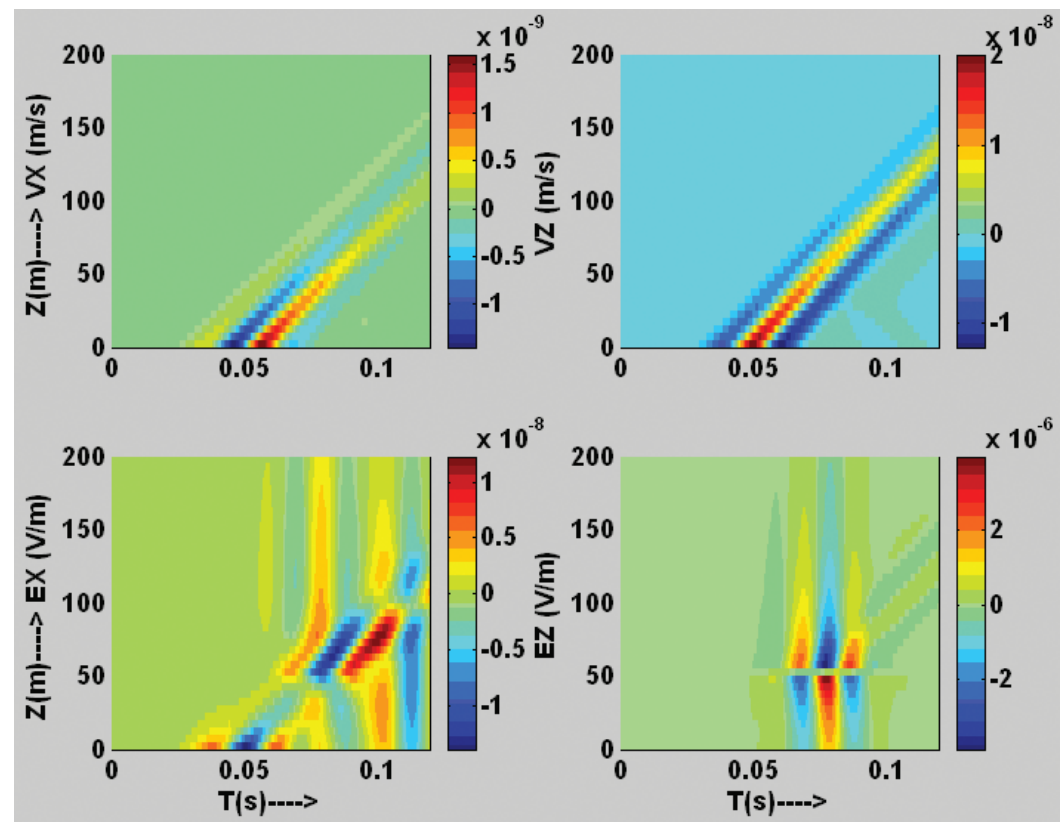

Figura 5 - Respostas sísmica (Vx e Vz) e sismoelétrica confinada-irradiada (Ex e Ez) devido ao contraste de porosidade, medidas na vertical nas direções $X$ e $Z$.

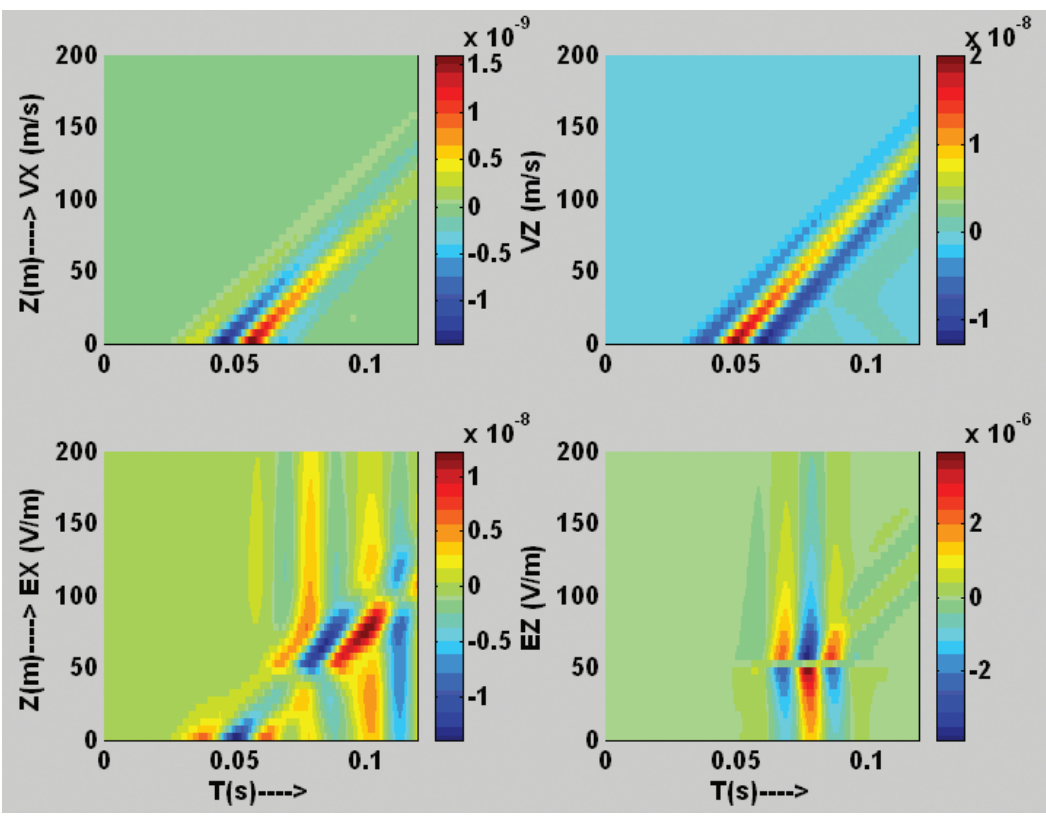

Figura 6 - Respostas sísmica (Vx e Vz) e sismoelétrica confinada-irradiada (Ex e Ez) devido ao contraste de salinidade, medidas na horizontal nas direções $X$ e Z.

salinidade. As concentrações usadas foram de 0.001 moles/litro para água limpa e 0.1 moles/litro para água contaminada. 0 potencial zeta e a condutividade do fluido foram calculados usando as fórmulas (43) e (44), respectivamente. A porosidade foi mantida constante e igual a $30 \%$.
A Figura 6 mostra as respostas sísmica (Vx e Vz) e sismoelétrica (Ex e EZ) medidas nas direções X e Z para o contraste de salinidade, com geometria de fonte e receptores baseada na Figura 3a. Observamos o sinal sismoelétrico confinado que acompanha o sinal sísmico e o sinal sismoelétrico irradiado si- 


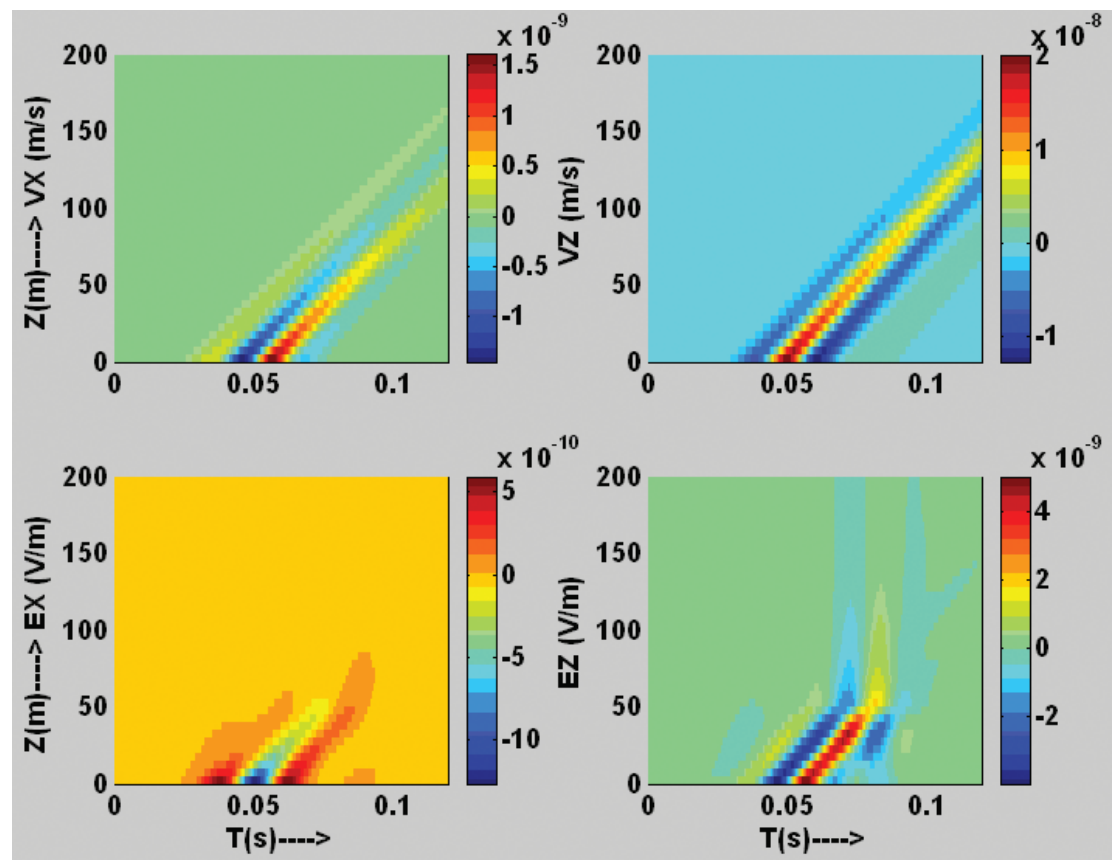

Figura 7 - Respostas sísmica (Vx e Vz) e sismoelétrica confinada-irradiada (Ex e Ez) devido ao contraste de salinidade, medidas na vertical nas direções $X$ e $Z$.

multâneo em todos os canais no tempo de 0.05 s. 0 sinal sismoelétrico confinado tem amplitude menor do que 0 sinal sismoelétrico irradiado cujos valores da ordem nanovolts por metro podem ser perfeitamente detectados. 0 sinal sísmico observado é a onda direta.

A Figura 7 mostra as respostas sísmica ( $V x$ e $V z)$ e sismoelétrica (Ex e EZ) medidas nas direções $X$ e $Z$ para 0 contraste de salinidade, com geometria de fonte e receptores baseada na Figura 3b. Observamos o sinal sismoelétrico confinado que acompanha o sinal sísmico, quase desaparece na região de alta salinidade, e o sinal sismoelétrico irradiado simultâneo em todos os canais e mais perceptível na direção Z. Os sinais sismoelétricos apresentam valores da ordem nanovolts por metro nos canais mais distantes e podem ser perfeitamente detectados. Não é observada nenhuma reflexão sísmica devido ao contraste de salinidade nos sismogramas indicando a insensibilidade da sísmica a este tipo de heterogeneidade.

Desta forma, um meio poroso saturado com fluidos que apresentem contraste de salinidade de 0.1 e 0.001 moles por litro, comuns em situações reais, pode gerar sinais sismoelétricos de amplitudes detectáveis e possíveis de serem mapeadas. 0 fato dos receptores sísmicos não detectarem o contraste de salinidade e os receptores elétricos detectarem o sismoelétrico irradiado no contraste de salinidade para a mesma fonte, é uma comprovação da complementaridade destes sinais. Outra observação impor- tante é que a diminuição da amplitude do sinal sismoelétrico confinado na região de mais alta salinidade pode ser usado em levantamento time lapse para monitorar frente de avanço de poluentes ambientais, com a vantagem do poder de investigar profundidades maiores devido ao confinamento ao sinal sísmico. 0 sinal sismoelétrico irradiado sofre variações de amplitude com a variação da salinidade como em Haines (2004) e pode ser usado para monitoramento time lapse em situações de objetivos rasos ou a pequenas distâncias dos receptores.

\section{Modelo de contato óleo-água}

A razão para este modelo foi a possibilidade da utilização da ferramenta em reservatórios petrolíferos para identificação do contato óleo-água devido ao contraste de condutividade elétrica, ao contraste de permissividade dielétrica, ao contraste de viscosidade, e ao contraste de densidade. Estes parâmetros aparecem implicitamente ou explicitamente no coeficiente de acoplamento eletrocinético e variam fortemente no contato óleo-água, cujos valores usados neste modelo foram: condutividade do óleo $10^{-5} \mathrm{~S} / \mathrm{m}$, condutividade da água $10^{-2} \mathrm{~S} / \mathrm{m}$, permissividade elétrica do óleo de $\varepsilon_{\text {Óleo }}=2 \varepsilon_{0}$, permissividade elétrica da água $\varepsilon_{\text {Áqua }}=80 \varepsilon_{0}$, viscosidade do óleo $\eta=0.1$ Pas, viscosidade da água $\eta=0.001$ Pas, densidade do óleo $\rho_{\text {Óleo }}=$ $800 \mathrm{~kg} / \mathrm{m}^{3}$, e densidade da água $\rho_{\text {Água }}=1000 \mathrm{~kg} / \mathrm{m}^{3}$. Supôs- 


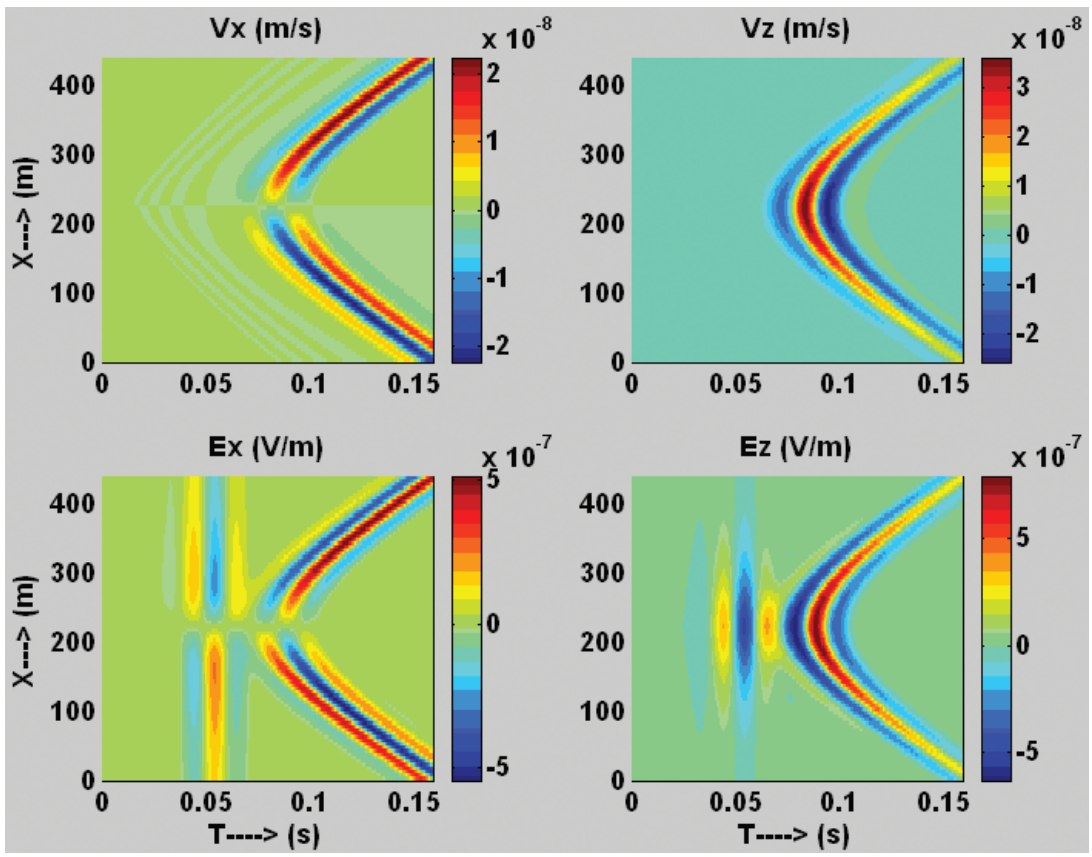

Figura 8 - Respostas sísmica (Vx e Vz) e sismoelétrica confinada-irradiada (Ex e Ez) devido ao contraste no contato óleo-água, medidas na horizontal nas direções X e Z.

se que a saturação residual de água fosse a mesma tanto na região de água quanto de óleo, o que fez usarmos o mesmo valor do potencial zeta nas duas regiões de fluido saturante, igual a $\zeta=0.065 \mathrm{~V}$.

A Figura 8 mostra as respostas sísmica ( $\mathrm{Vx}$ e $\mathrm{Vz}$ ) e sismoelétrica (Ex e Ez) medidas nas direções $X$ e $Z$ para 0 contato óleo-água, com geometria de fonte e receptores baseada na Figura 3a. Observamos o sinal sismoelétrico confinado que acompanha o sinal sísmico e o sinal sismoelétrico irradiado simultâneo em todos os canais no tempo de aproximadamente $0.05 \mathrm{~s}$. 0 sinal sismoelétrico confinado tem amplitude similar ao sinal sismoelétrico irradiado com valores da ordem de centenas de nanovolts por metro perfeitamente detectável. 0 sinal sísmico observado é a onda direta.

A Figura 9 mostra as respostas sísmica ( $V x$ e Vz) e sismoelétrica (Ex e EZ) medidas nas direções X e Z para 0 contato óleo-água, com geometria de fonte e receptores baseada na Figura 3b. Observamos o sinal sismoelétrico confinado que acompanha o sinal sísmico e o sinal sismoelétrico irradiado simultâneo em todos os canais no tempo de $0.8 \mathrm{~s}$ aproximadamente. 0 sinal sismoelétrico confinado tem amplitude similar ao sinal sismoelétrico irradiado com valores da ordem de centenas de nanovolts por metro perfeitamente detectável. Notamos também que existe uma reflexão sísmica muito fraca devido ao contato óleo-água.
Os resultados das medidas sísmicas e sismoelétricas do modelo de contato óleo-água mostrados nas Figuras 8 e 9 indicam respostas mensuráveis e possibilidade do uso do sinal sismoelétrico irradiado para mapear a superfície de contato óleoágua em reservatórios petrolíferos conjuntamente com levantamentos de sísmica de poço e tomografia entre poços. 0 sinal sismoelétrico forte observado no contato óleo-água pode ser usado com informação adicional à fraca reflexão sísmica.

\section{Modelo de contraste litológico}

0 modelo de contraste litológico se reflete no contraste dos parâmetros elásticos, da porosidade, da permeabilidade, e da condutividade. 0 modelo de contraste litológico investigado foi 0 da Tabela 1. 0 valor do potencial zeta no Arenito e no Folhelho (Eq. (43)), igual a $\zeta=0.065 \mathrm{~V}$.

A Figura 10 mostra as respostas sísmica ( $V x$ e Vz) e sismoelétrica (Ex e EZ) medidas nas direções $X$ e $Z$ para 0 modelo de contraste litológico, com geometria de fonte e receptores baseada na Figura 3a. Observamos o sinal sismoelétrico confinado que acompanha o sinal sísmico e o sinal sismoelétrico irradiado simultâneo em todos os canais no tempo aproximado de $0.05 \mathrm{~s}$. 0 sinal sismoelétrico confinado tem amplitude similar ao sinal sismoelétrico irradiado com valores da ordem de dezenas de milivolts por metro absolutamente detectável. 0 sinal sísmico observado é a onda direta. 


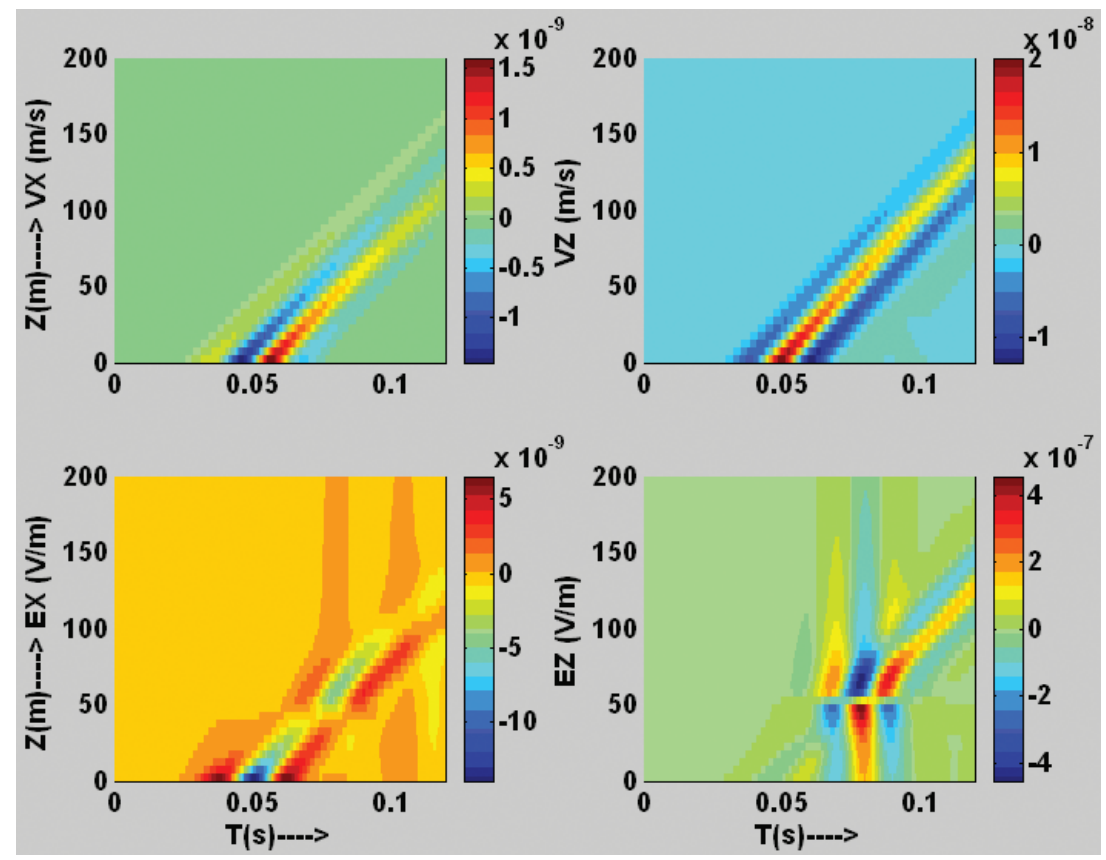

Figura 9 - Respostas sísmica (Vx e Vz) e sismoelétrica confinada-irradiada (Ex e Ez) devido ao contraste no contato óleo-água, medidas na vertical nas direções X e Z.

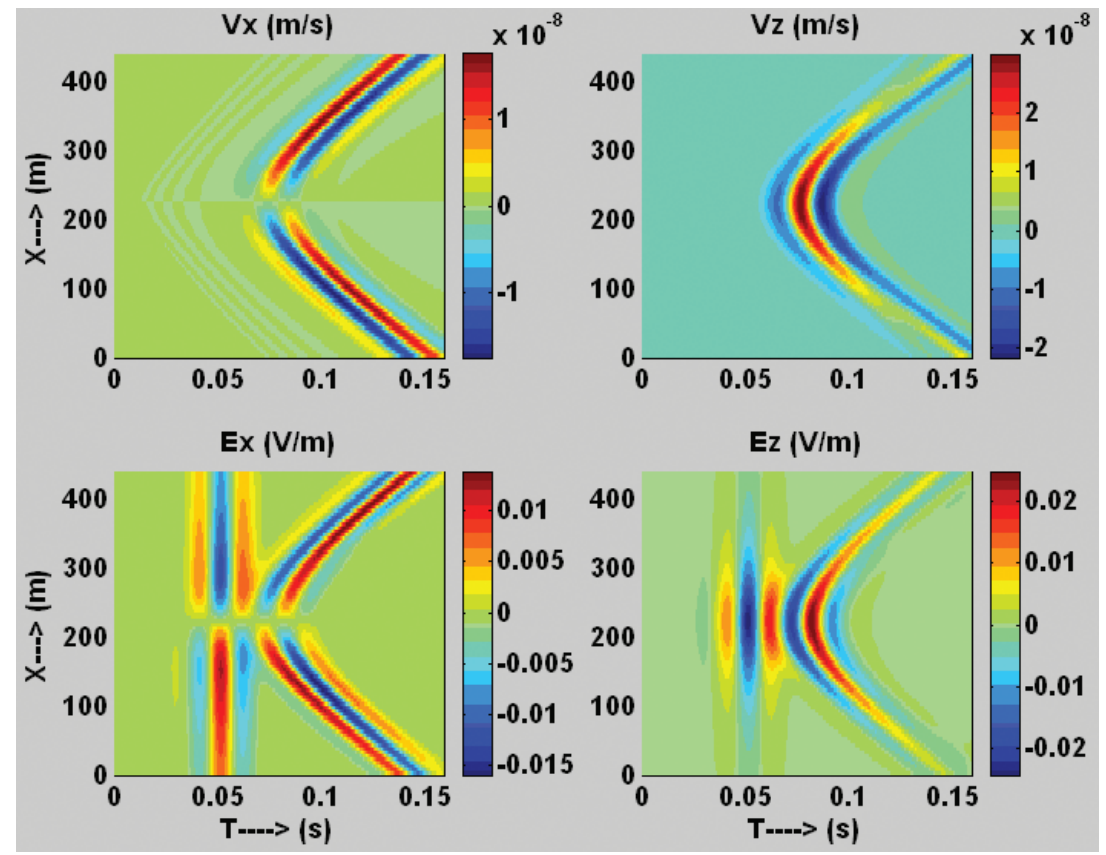

Figura 10 - Respostas sísmica (Vx e Vz) e sismoelétrica confinada-irradiada (Ex e Ez) devido à interface arenitoargila, medidas na horizontal nas direções $\mathrm{X}$ e Z.

A Figura 11 mostra as respostas sísmica ( $\mathrm{Vx}$ e $\mathrm{Vz})$ e sismoelétrica (Ex e EZ) medidas nas direções $X$ e $Z$ para o modelo de contraste litológico, com geometria de fonte e receptores ba- seada na Figura 3b. Observamos o sinal sismoelétrico confinado que acompanha o sinal sísmico e o sinal sismoelétrico irradiado simultâneo em todos os canais no tempo de 0.08 s aproxima- 
damente. 0 sinal sismoelétrico confinado tem amplitude similar ao sinal sismoelétrico irradiado com valores da ordem de centenas de milivolts por metro absolutamente detectável. Notamos também que existe uma reflexão sísmica muito boa no contato arenito-argila.

A resposta sismoelétrica irradiada do contraste litológico arenito-argila apresenta 0 maior valor absoluto de amplitude da ordem de centenas de milivolts, causado pelo contraste do grande número de parâmetros sismoelétricos (parâmetros poroelásticos e elétricos). Adicionalmente ao mapeamento de interfaces litológicas, com uso combinado do sinal sismoelétrico irradiado com reflexões sísmicas e dos parâmetros poroelásticos pode-se inferir a condutividade elétrica e conseqüentemente 0 tipo do fluido saturante. Também, as medidas simultâneas do sinal sismoelétrico confinado e das reflexões sísmicas contém informações sobre a condutividade do fluido saturante (Garambois \& Dietrich, 2001).

\section{Modelo de detectabilidade de heterogeneidade}

0 modelo investiga a detectabilidade de uma camada fina de argila de $5 \mathrm{~m}$ de espessura, encaixada num arenito poroso. Os parâmetros deste modelo são os mesmos do arenito e da argila da Tabela 1 usados no modelo de contraste litológico anterior.

A Figura 12 representa 0 modelo que investiga a detectabilidade de uma camada fina de argila encaixada num arenito poroso. A Figura 12a representa a geometria de aquisição sismoelétrica com receptores dispostos na horizontal e a Figura 12b representa a aquisição na vertical.

A Figura 13 mostra as respostas sísmica (Vx e Vz) e sismoelétrica (Ex e EZ) medidas nas direções X e Z para o modelo de detecção de camada fina, com geometria de fonte e receptores baseada na Figura 12a. Observamos na figura que 0 sinal sismoelétrico confinado quase desaparece em relação ao forte sinal sismoelétrico irradiado que ocorre simultaneamente no tempo de $0.05 \mathrm{~s}$, com amplitude da ordem de centenas de milivolts por metro. A redução de amplitude do sinal sismoelétrico confinado em relação ao sinal sismoelétrico irradiado é devido à porosidade alta do arenito background.

A Figura 14 mostra as respostas sísmica (Vx e Vz) e sismoelétrica ( $E x$ e EZ) medidas nas direções $X$ e Z para o modelo de detecção de camada fina, com geometria de fonte e receptores baseada na Figura 12b. Observamos na figura que 0 sinal sismoelétrico confinado Ex e Ez quase desaparece em relação ao forte sinal sismoelétrico irradiado que ocorre simultaneamente no tempo de $0.075 \mathrm{~s}$. A redução de amplitude do sinal sismo- elétrico confinado em relação ao sinal sismoelétrico irradiado é devido à porosidade alta do arenito background.

0 sinal sismoelétrico irradiado da Figura 14 detecta perfeitamente a camada fina de $5 \mathrm{~m}$ de espessura do modelo estudado enquanto o sinal sísmico detecta como uma reflexão que se desprende da onda direta no tempo de $0.8 \mathrm{~s}$. 0 que se observa nas Figuras 13 e 14 é que uma camada fina de 5 m é capaz de gerar sinal sismoelétrico irradiado com amplitude detectável indicando o seu potencial para mapear camadas finas como os folhelhos que causam barreiras de fluxo em reservatórios de água e petróleo.

Acredita-se que seja possível detectar camadas bem mais finas devido ao fato de que a irradiação do campo elétrico difusivo seja causada por qualquer heterogeneidade que desacelere as cargas elétricas separadas pelo pulso sísmico como lâminas de $0.5 \mathrm{~m}$, por exemplo. Para a espessura de $5 \mathrm{~m}$ da heterogeneidade do modelo estudado ainda ocorre um sinal sísmico refletido que certamente desaparecerá para espessuras menores.

No geral observa-se que dentre os sinais irradiados, existe uma diferença de amplitude entre eles. A amplitude do campo elétrico irradiado varia conforme o tipo de contraste:

1. 0 campo elétrico mais forte é gerado pelo contraste litológico arenito-argila, da ordem de $10^{-2} \mathrm{~V} / \mathrm{m}$. 0 modelo de camada fina, devido ao mesmo contraste apresenta amplitude da mesma ordem de grandeza.

2. 0 campo elétrico gerado pelo contraste de porosidade é da ordem de $10^{-6} \mathrm{~V} / \mathrm{m}$.

3. 0 campo elétrico gerado pelo contato óleo-água é da ordem de $10^{-7} \mathrm{~V} / \mathrm{m}$.

4. 0 campo elétrico gerado pelo contraste de salinidade é da ordem de $10^{-9} \mathrm{~V} / \mathrm{m}$.

\section{CONCLUSÃO}

A solução numérica pelo método das diferenças finitas das equações sismoelétricas apresentada neste trabalho obteve as respostas sismoelétricas da simulação do contraste de heterogeneidades de um modelo poroso granular constituído de arenito e argila saturados, cujas propriedades físicas são conhecidas.

A principal conclusão qualitativa é que todos os modelos estudados apresentam resposta sismoelétrica irradiada e confinada.

A principal conclusão quantitativa é que dentre os sinais irradiados, existe uma diferença de amplitude entre eles. A amplitude do campo elétrico irradiado varia conforme o tipo de contraste: 


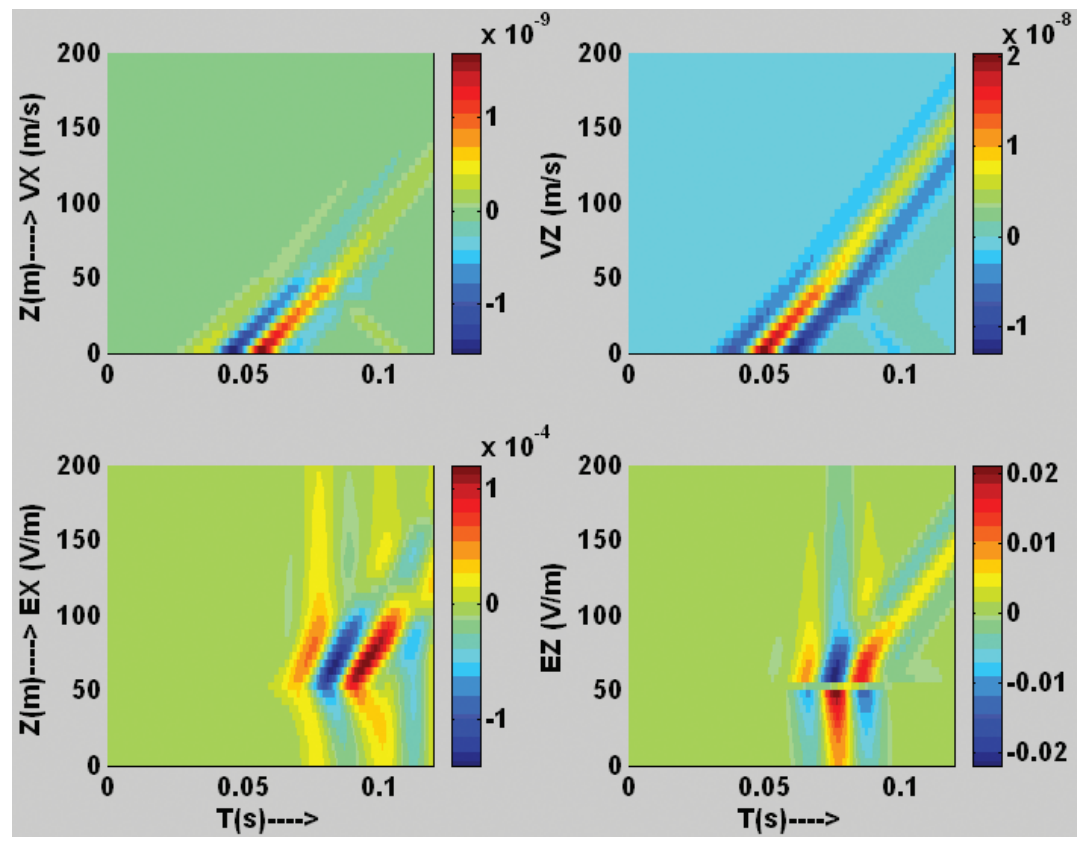

Figura 11 - Respostas sísmica (Vx e Vz) e sismoelétrica confinada-irradiada (Ex e Ez) devido à interface arenitoargila, medidas na vertical nas direções $X$ e $Z$.
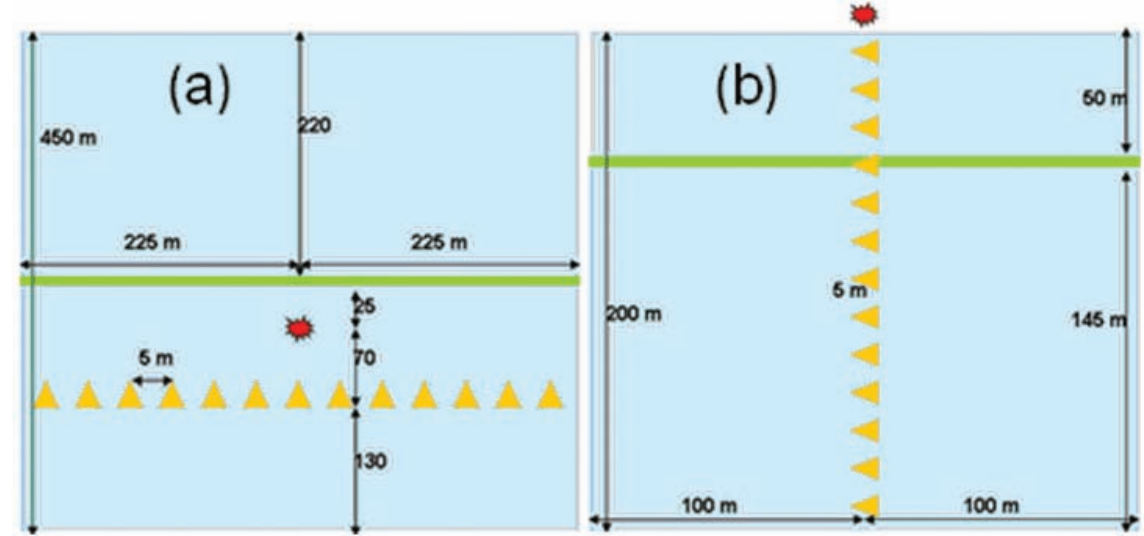

Figura 12 - Geometrias de fonte e receptores usadas nos modelos de camada fina. A figura (a) simula receptores na horizontal e a figura (b) na vertical.

1. 0 campo elétrico mais forte é gerado pelo contraste litológico are-nito-argila, da ordem de $10^{-2} \mathrm{~V} / \mathrm{m}$. 0 modelo de camada fina, devido ao mesmo contraste apresenta amplitude da mesma ordem de grandeza.

2. 0 campo elétrico gerado pelo contraste de porosidade é da ordem de $10^{-6} \mathrm{~V} / \mathrm{m}$.

3. 0 campo elétrico gerado pelo contato óleo-água é da ordem de $10^{-7} \mathrm{~V} / \mathrm{m}$.

4. 0 campo elétrico gerado pelo contraste de salinidade é da ordem de $10^{-9} \mathrm{~V} / \mathrm{m}$.
Provavelmente em situações reais os contrastes litológicos serão mais detectáveis do que os contrastes de porosidade, fluido e salinidade. 0 fato de diferentes contrastes gerarem diferentes amplitudes sismoelétricas não garante a discriminação do tipo de contraste, pois existem outros fatores envolvidos na composição da amplitude como a distância da interface ao receptor e a potência da fonte, ruídos.

A resposta sismoelétrica ao contraste de porosidade evidencia a possibilidade do mapeamento de contraste de porosidade e a identificação de zonas mais propícias a reservatórios.

As respostas sismoelétricas de uma camada fina de argila 


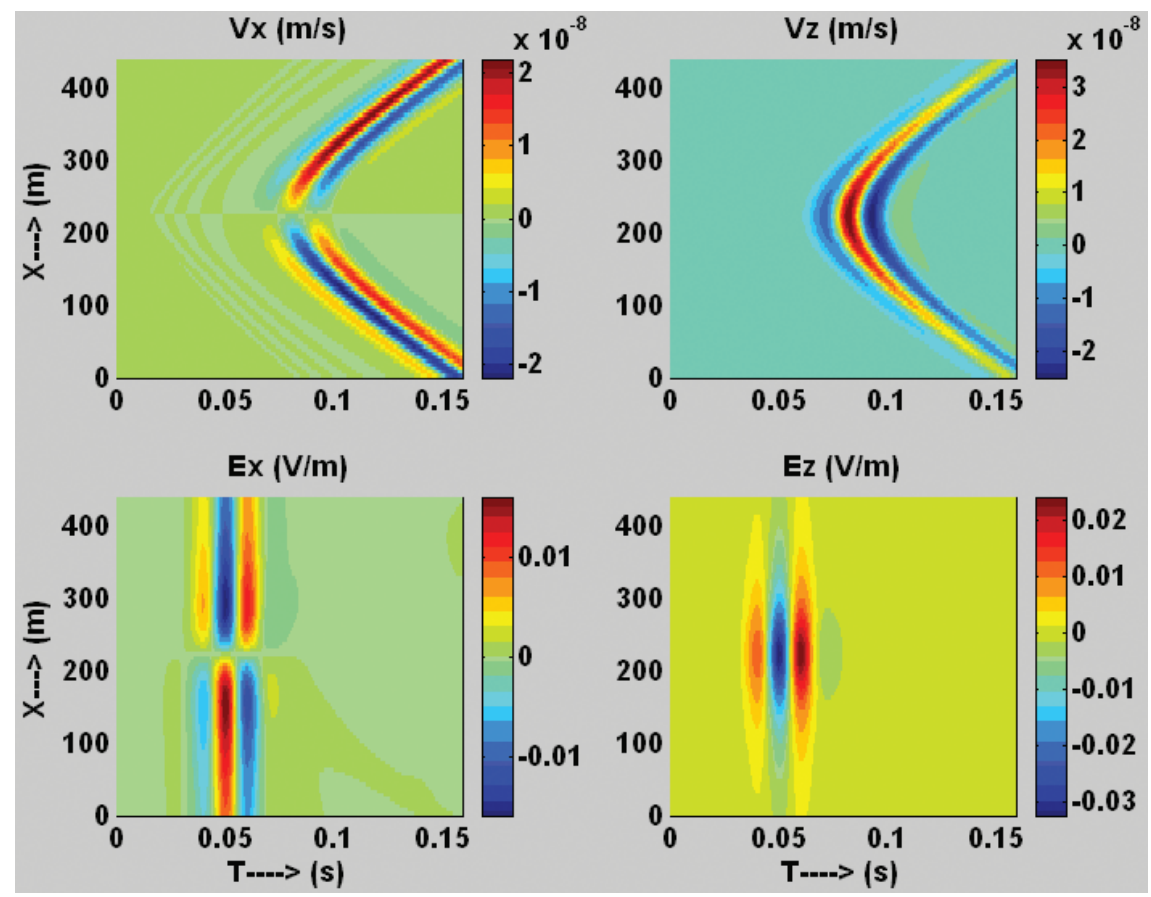

Figura 13 - Respostas sísmica (Vx e Vz) e sismoelétrica confinada-irradiada (Ex e Ez) devido a uma camada fina de $5 \mathrm{~m}$ de argila num arenito, medidas na horizontal nas direções $X$ e $Z$.

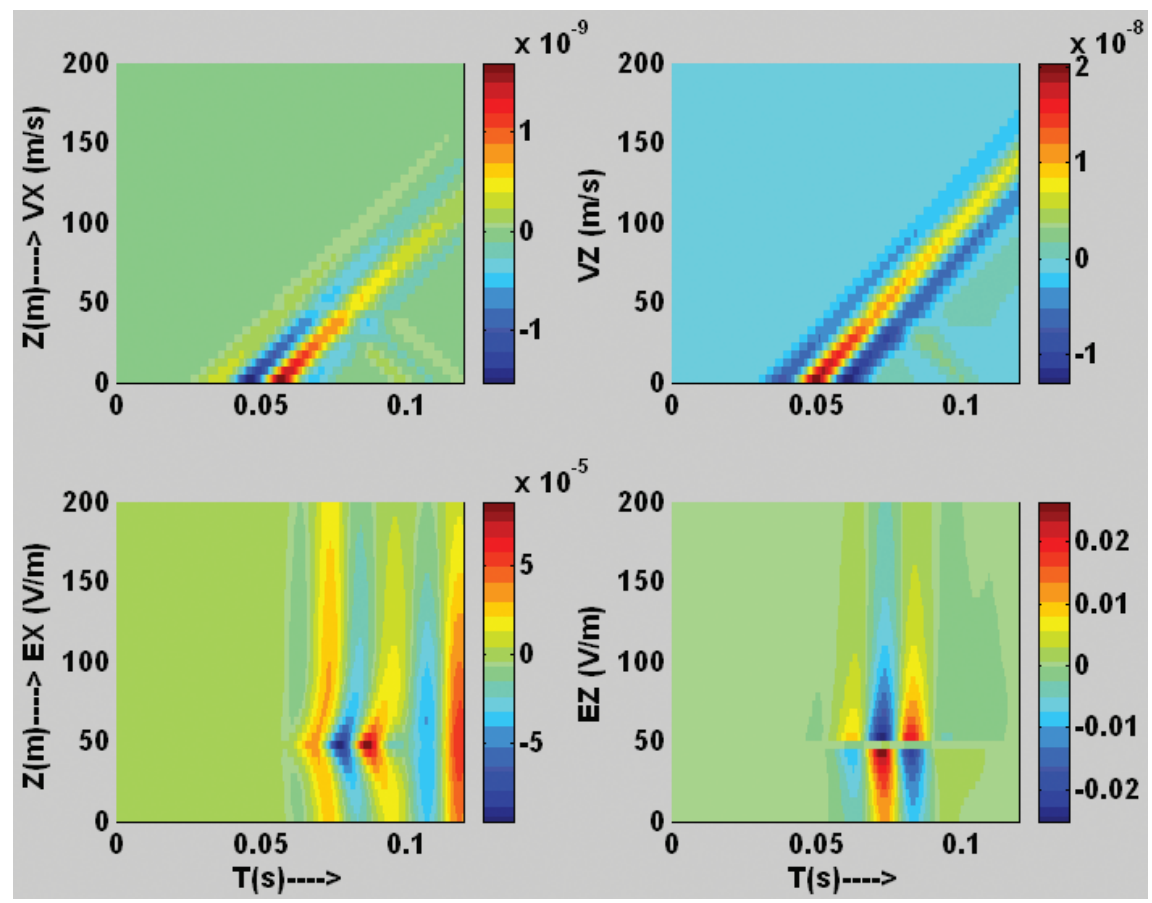

Figura 14 - Respostas sísmica (Vx e Vz) e sismoelétrica confinada-irradiada (Ex e Ez) devido a uma camada fina de $5 \mathrm{~m}$ de argila num arenito, medidas na vertical nas direções $X$ e $Z$. 
dentro de um arenito permitiram a definição das dimensões mínimas de detectabilidade de heterogeneidades. Estas dimensões coincidem com as dimensões da malha de diferenças finitas. Desta forma, as microestruturas com dimensões desta ordem de grandeza, como barreiras de fluxo e microfraturas, podem ser detectadas por ferramentas sismoelétricas. Uma solução do problema da estabilidade numérica para uso de uma malha mais fina permitiria um estudo mais detalhado sobre as dimensões mínimas de detectabilidade das heterogeneidades.

Este trabalho contribui para a modelagem numérica do sinal sismoelétrico em interfaces com contraste de porosidade, salinidade, litologia, contato óleo-água, para inferir a detectabilidade de microestruturas não detectadas pela sísmica.

A detecção de sinais sismoelétricos nos receptores dispostos na horizontal contribui para que se possa fazer uma aquisição sismoelétrica conjunta com a sísmica de superfície para objetivos rasos, e a detecção de sinais sismoelétricos nos receptores dispostos na vertical contribui para que se possa fazer uma aquisição sismoelétrica conjunta com a sísmica de poços (VSP) ou para uma aquisição tomográfica interpoços, dentro dos limites do raio de investigação.

A modelagem sismoelétrica em freqüências acima das freqüências sísmicas, nos caso sônico e ultrasônico, podem ser feitas para se testar a possibilidade de medidas sismoelétricas conjuntas com as perfilagens sônicas. Nesta banda de freqüências a poroelasticidade tem comportamento inercial.

A elaboração de uma ampla variedade de modelos sismoelétricos que contivesse mais complexidade seria importante para melhorar 0 entendimento das respostas sismoelétricas em ambientes complexos. Uma sugestão seria a construção de modelos sismoelétricos a partir de dados de poços como no caso de áreas petrolíferas. Por exemplo, construir modelos de rochas carbonáticas, de folhelhos, dos vários tipos de reservatórios. Também seria muito importante um estudo de laboratório de efeitos eletroquímicos dos dados de poços como o potencial zeta, a condutividade, efeitos dos fluidos.

A generalização da solução numérica do acoplamento poroelástico-eletromagnético para o caso eletrosísmico (neste caso a fonte é a corrente elétrica) seria importante para estudar os efeitos da conversão da energia elétrica para energia sísmica. Uma vantagem do método eletrosísmico é a redução do ruído pela utilização de fontes de correntes que usam longas séries temporais (semelhante aos vibradores) e a utilização de receptores de estado sólido como os acelerômetros.

0 desenvolvimento de programas para inversão dos dados sismoelétricos poderá nos levar à estimativa das propriedades físicas do fluido e das propriedades físicas e petrofísicas do esqueleto poroso.

\section{AGRADECIMENTOS}

Os autores agradecem ao LENEP/UENF pelo apoio no desenvolvimento deste trabalho. E também aos revisores anônimos pelas críticas e sugestões que contribuíram para finalização deste artigo.

\section{REFERÊNCIAS}

ANDRÉ ABQ. 2005. Modelagem sísmica em meios poroelásticos heterogêneos: Dissertação de mestrado, LENEP/UENF, Macaé, RJ, Brasil. $87 \mathrm{pp}$.

BERRYMAN JG. 1980. Confirmation of Biot's theory. Appl. Phys. Lett., 37: 382-384.

BUTLER KE, FLEMING SW \& RUSSELL RD. 1999. Field test for linearity of seismoelectric conversions. Canadian Journal of Exploration Geophysics, 35: 20-23.

BUTLER KE, RUSSELL RD, KEPIC AW \& MAXWELL M. 1996. Measurement of the seismoelectric response from a shallow boundary. Geophysics, 61(6): 1769-1778.

CARCIONE JM \& QUIROGA-GOODE G. 1995. Some aspects of the physics and numerical modeling of Biot compressional waves. Journal of Computational Acoustics, 3(4): 261-280.

CARCIONE JM. 2001. Wave fields in real media. Wave propagation in anisotropic, anelastic and porous media. Elsevier, NY, 370 pp.

CERJAN C, KOSLOFF D, KOSLOFF R \& RESHEF M. 1985. A nonreflecting boundary condition for discrete acoustic and elastic wave equations. Geophysics, 50(4): 705-708.

CUMINATO JA \& MENEGUETTE Jr M. 2002. Discretização de Equações Diferenciais Parciais - Técnica de Diferenças Finitas. Notas de Aula, UFSC.

DUPUIS JC, BUTLER KE \& KEPIC AW. 2007. Seismoelectric imaging of the vadose zone of a sand aquifer. Geophysics, 72(6): A81-A85.

FOURIE FD. 2003. Application of Electroseismic Techniques to Geohydrological Investigations in Karoo Rocks. PhD Thesis, University of the Free State, 177 pp.

GARAMBOIS S \& DIETRICH M. 2001. Seismoelectric wave conversions in porous media: Field measurements and transfer function analysis. Geophysics, 66(5): 1417-1430.

HAINES SS. 2004. Seismoelectric imaging of shallow targets. PhD Thesis, Stanford University, $179 \mathrm{pp}$.

LEVANDER AR. 1988. Fourth-order finite-difference $P-S V$ seismograms. Geophysics, 53(11): 1425-1436. 
MANDARIAGA R. 1976. Dynamics of an expanding circular fault. Bull. Seis. Soc. Am., 66: 639-666.

MAVKO G, TAPAN M \& DVORKIN J. 1998. The Rock Physics Handbook: Tools for Seismic Analysis in Porous Media. Cambridge University Press, NY, USA. 329 pp.

MIKHAILOV OV, HAARTSEN MW \& TOKSÖZ MN. 1997. Electroseismic investigation of the shallow subsurface: Field measurements and numerical modeling. Geophysics, 62(1): 97-105.

MIKHAILOV OV, QUEEN J \& TOKSÖZ MN. 2000. Using borehole electroseismic measurements to detect and characterize fractured (permeable) zones. Geophysics, 65(4): 1098-1112.

MURTY Y. 1985. First results on the direct detection of groundwater by seismoelectric effect $-\mathrm{a}$ field experiment. Bull. Aust. Soc. Expl. Geophys., 16: 254-255.

PRIDE SR. 1994. Governing equations for the coupled electromagnetics and acoustic of porous media. Physical Review B, 50(21): 15678-15696.

PRIDE SR \& MORGAN D. 1991. Electrokinetic dissipation induced by seismic waves. Geophysics, 56(7): 914-925.

PRIDE SR, TROMEUR E \& BERRYMAN JG. 2002. Biot slow-wave effects in stratified rocks. Geophysics, 67(1): 271-281.

SANTOS LA, BULCÃO A \& SOARES FILHO DM. 2006. Modelagem sísmica em meios porosos - Rotina P0R02D, Relatório Técnico 001/2006, CENPES-PETROBRAS. 59 pp.
STRAHSER M, IWANOWSKI K \& RABBEL W. 2007. Vertical seismoelectric profiling - dependence on hydrogeological parameters. Geophysical Research Abstracts, 9.

THOMPSON AH \& GIST GA. 1993. Geophysical applications of electrokinetic conversion. The Leading Edge, 12: 1169-1173.

VIRIEUX J. 1984. $S H$-wave propagation in heterogeneous media: Velocity-stress finite-difference method. Geophysics, 49(11): 19331957.

VIRIEUX J. 1986. $P-S V$ wave propagation in heterogeneous media: Velocity-stress finite-difference method. Geophysics, 51(4): 889-901.

WYLLIE MRJ, GREGORY AR \& GARDNER LW. 1956. Elastic wave velocities in heterogeneous and porous media. Geophysics, 21: 41-70.

YEE KS. 1966. Numerical solution of initial boundary value problems involving Maxwell's equations in isotropic media. IEEE Trans. Antennas and Propagation, vol. AP-4(3): 302-307.

ZHU Z \& TOKSÖZ MN. 2003. Crosshole seismoelectric measurements in borehole models with fractures. Geophysics, 68(5): 1519-1524.

ZHU Z \& TOKSÖZ MN. 2005. Seismoelectric and seismomagnetic measurements in fractured borehole models. Geophysics, 70(4): 45-51.

ZHU Z, HAARTSEN MW \& TOKSÖZ MN. 1999. Experimental studies of electrokinetic conversions in fluid-saturated borehole models. Geophysics, 64(5): 1349-1356.

\section{NOTAS SOBRE OS AUTORES}

Francisco Joclean Alves Vanzeler é graduado em Física pela Universidade Federal do Pará (UFPA), em 1984. Obteve o seu título de Mestre em Geofísica pela Universidade Estadual de Campinas (UNICAMP), em 2000. Obteve seu Doutorado em Geofísica pela Universidade Estadual do Norte Fluminense Darcy Ribeiro (UENF), em 2008. Atualmente trabalha com interpretação sísmica na Petrobras.

Viatcheslav Ivanovich Priimenko é graduado em Matemática Pura e Aplicada pela Universidade Federal de Novosibirsk (UFN), Akademgorodok, Novosibirsk, Rússia, em 1976. Obteve o seu título de Mestre em Matemática na UFN em 1978. Obteve seu Doutorado em Física-Matemática em 1990 na UFN. Obteve seu título de Livre Docente em 1997 na UFN. Atualmente é professor da UENF e chefe do Laboratório de Engenharia e Exploração de Petróleo (LENEP). Áreas de interesse: problemas diretos e inversos de geofísica e engenharia de petróleo, modelagem numérica e ensino nas áreas de matemática, geofísica e engenharia de petróleo. 